\title{
Kinetic Studies of Gas Flows
}

\author{
Jing Fan* and Quanhua Sun \\ LHD, Institute of Mechanics, Chinese Academy of Sciences, Beijing 100190, PRC \\ (*jfan@imech.ac.cn)
}

\begin{abstract}
Our recent studies on kinetic behaviors of gas flows are reviewed in this paper. These flows have a wide range of background, but share a common feature that the flow Knudsen number is larger than 0.01 . Thus kinetic approaches such as the direct simulation Monte Carlo method are required for their description. In the past few years, we studied several micro/nano-scale flows by developing novel particle simulation approach, and investigated the flows in low-pressure chambers and at high altitude. In addition, the microscopic behaviors of a couple of classical flow problems were analyzed, which shows the potential for kinetic approaches to reveal the microscopic mechanism of gas flows.
\end{abstract}

Keywords: Kinetic Approach, Micro/Nano Flow, Vacuum Chamber, Hypersonic Flow, DSMC Method, IP Method. PACS: 47.45.-n; 05.70.Ln; 05.10.Ln

\section{INTRODUCTION}

Kinetic effects in gas flows have been paid more and more attention in many fields such as the rapidly developing micro-electro-mechanical systems, material and vacuum systems, and the traditional high-altitude, highspeed vehicles [1]. The common feature in these applications is that the classical continuum assumption is inaccurate or invalid to describe the related flows because the Knudsen (Kn) number, the ratio of the mean free path to the characteristic flow length, is not small enough to be neglected. In general, the flow is continuum when $K n<0.01$; thereafter the flow will have obvious velocity slip or temperature jump on a wall surface, and continuum equation may be still used with proper treatment on wall boundaries for the Knudsen number up to 0.1; when $0.1<K n<10$, the flow is in the transitional regime, where inter-molecular collisions and molecular-surface collisions are equally important; when $K n>10$, the inter-molecular collisions become unimportant, and the flow can be described by the free-molecular theory.

In the kinetic viewpoint, the general governing equation for gas flows is the Boltzmann equation that describes the time evolution of the velocity distribution function [2]. This equation is a seven-dimensional integro-differential equation that includes a complicated collision term, which is very difficult to be solved directly. Thus model equations such as the BGK equation are sometimes employed instead. Another kinetic approach is to track particle trajectories such as the direct simulation Monte Carlo (DSMC) method [3] that has achieved great success in simulating various rarefied flows. In the DSMC method, a large number of represented particles are used to mimic the microscopic motions of real gas molecules, where inter-collision motions are treated deterministically and particle collisions are handled statistically. The computational cost for a DSMC simulation, in general, is much less than the cost for a molecular dynamics (MD) simulation, so the former is more suitable to simulate gas flows than the later.

In this paper, we will review our kinetic studies of gas flows in recent years and show some examples to reflect current progress.

\section{MICRO/NANO-SCALE FLOWS}

Micro- and nano-scale flows are becoming popular because of the rapid progress of the micro- or nano- scale systems. The small length scale of these systems usually leads to a Knudsen number larger than 0.001 even at the atmospheric condition. Many numerical approaches have been applied to study these flows, including Navier-Stokes solvers using slip boundary conditions and schemes solving the lattice Boltzmann equation. The DSMC method [3], as a popular particle approach, has also been applied for some MEMS flow simulations. But the low Mach number flows encountered in MEMS/NEMS make a DSMC simulation very time-consuming in order to reduce the statistical noise. The information preservation (IP) method [4] was then proposed and can successfully reduce the statistical scatter in a DSMC simulation.

\footnotetext{
CP1233 The $2^{\text {nd }}$ International ISCM Symposium and The $12^{\text {th }}$ International EPMESC Conference

J. W. Z. Lu, A.Y.T. Leung, V. P. Iu, and K. M. Mok

(C) 2010 American Institute of Physics 978-0-7354-0778-7/10/\$30.00
} 


\section{Information Preservation Method}

In the kinetic viewpoint, fluid flows are determined by molecular motion under specified initial and boundary conditions. The IP method [4] initially assigns each simulated molecule an IP velocity and temperature based on the initial conditions of a problem interested, and then they transport along with the molecular motion. When a simulated molecule collides with another, the two exchange the IP quantities according to the IP collision models; when the molecule encounters a computational boundary during its movement, the IP velocity and temperature take the boundary values. The flow field is obtained through sampling and averaging the IP velocity and temperature that avoid the computational noise arising from directly sampling and averaging molecular thermal motions.

The IP method was originally proposed to solve micro gas flows where the motions of simulated molecules can be described well by the DSMC methods [4]. Because the transport processes of the IP quantities are carried out directly through molecular motions, the IP method works over the entire Knudsen regime from continuum to free molecular. The IP method can also be extended to other situations such as liquid using the molecular dynamics (MD) instead of DSMC to calculate molecular trajectories.

The IP method was firstly applied to several unidirectional transitional gas flows with a wide range of Knudsen number. It was then developed to include density variation [7] and temperature change [8]. New applications of the method include the micro-channel flows [5], flat plate aerodynamics [6], and 3D flows in read-write head slider [9], which showed excellent agreement with measured data available for microchannel and flat plates.

Another important advancement was made by Sun and Boyd who found that the information preserved in the IP method can be tracked using the Maxwell's transport equation and derived the governing equations for the preserved information [10]. They also showed that the previous schemes could be directly derived from the governing equations. Masters and Ye then extended the idea and developed the octant flux splitting scheme where they found that the splitting direction for fluxes was important [11]. These studies not only solved some hard problems but also enriched the content of the IP method.

\section{Micro-Scale Flow Simulations}

Recent applications of the IP method include the low-speed internal and external microscale flow simulations. For low Reynolds number flows, the kinetic effects can be important because the Knudsen number is related to the Mach number and the Reynolds number $(K n=M a / R e)$.

Figure 1 shows some simulation results for lid-driven cavity flows when the Reynolds number is 10 . At this Reynolds number, the Mach number effects are unimportant for low-speed flows as predicted by the Navier-Stokes solutions especially for the streamlines. However, when the kinetic effects are included, the streamline patterns have obvious changes with the flow Mach number or the Knudsen number. Specifically, when the Knudsen number increases with the Mach number, the corner vortexes shrink or even disappear. This behavior is also observed for flows over a micro-cylinder as shown in Fig. 2. The results in Fig. 2 are obtained using a hybrid continuum-particle approach that combines a Navier-Stokes solver and the IP method, and that the IP method is applied only to a small domain around the cylinder.
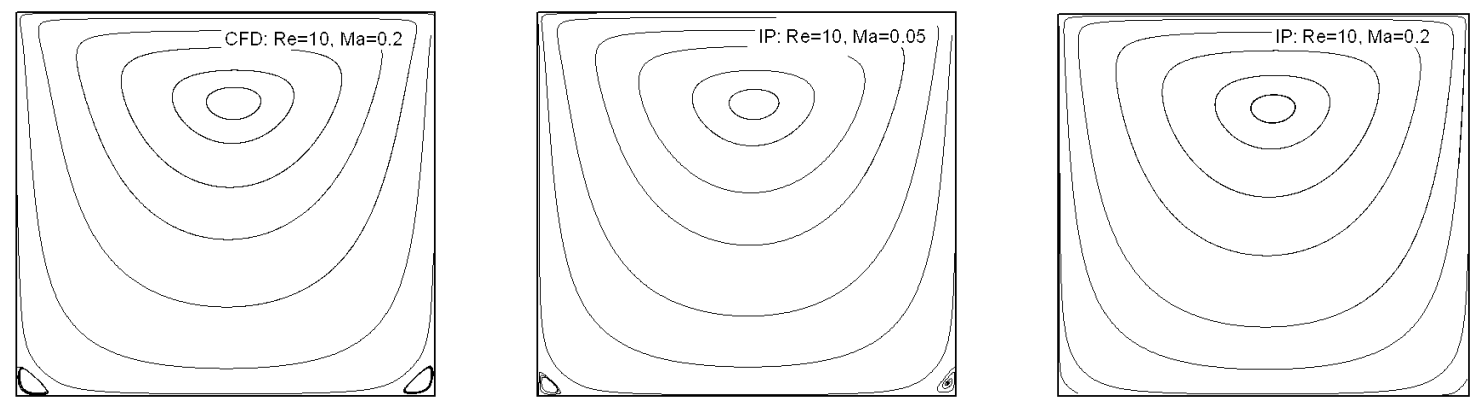

FIGURE 1. Streamlines in the lid-driven cavity flows when the Reynolds number is 10. Left: Navier-Stokes results for Ma=0.2; middle: IP results for $\mathrm{Ma}=0.05$ where $\mathrm{Kn}=0.006$; right: IP results for $\mathrm{Ma}=0.2$ where $\mathrm{Kn}=0.025$.
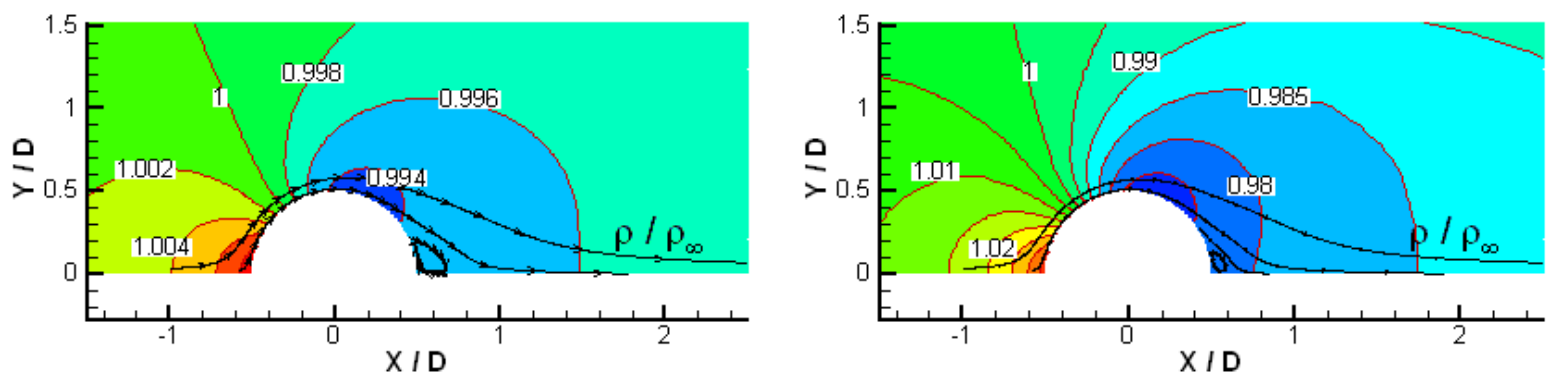

FIGURE 2. Density and streamlines for flow over a cylinder with $\mathrm{D}=20$ microns. Left: $\mathrm{Re}=10, \mathrm{Ma}=0.1$; right: $\mathrm{Re}=10, \mathrm{Ma}=0.2$. 
It is usually very difficult to find a simple expression to quantify the kinetic effects for flows. However, for lowspeed flow over a flat plate, we obtain a satisfactory expression using our IP results and the experimental data in the literature. It is known that the drag coefficient on the plate for incompressible flow depends only on the Reynolds number whereas the free molecular results depend only on the Mach number. The general expression is the combination of the two expressions at the limit conditions, which is shown in equation (1).

$$
C_{D}=\frac{C_{D, \text { continuum }} \cdot C_{D, \text { free molecular }}}{C_{D, \text { continuum }}+C_{D \text {, free molecular }}}
$$

where, $C_{D, \text { continuum }}=2.656 / \sqrt{\mathrm{Re}}+10.6 / \mathrm{Re}$, and $C_{D, \text { free molecular }}=1.349 / \mathrm{Ma}$. The accuracy of this expression is very good for the whole range of the Knudsen number.

\section{LOW-PRESSURE FLOWS IN VACUUM CHAMBER}

Another kind of non-continuum flows occurs in vacuum chambers where the gas pressure is very low. Vacuum chamber can provide clean environment for material processing or mimic the outer space environment.

For material processing, we have developed a multi-electron-beam physical vapor deposition (EBPVD) system that is illustrated in Fig. 3 [12]. The main body of the system is a cylindrical vacuum chamber whose diameter is $760 \mathrm{~mm}$ and the height is $850 \mathrm{~mm}$. Equipments installed inside include three sets of electron guns and evaporation crucibles and one set of electric resistance evaporation equipment. The rate of each evaporation source is monitored and controlled by the corresponding quartz crystal probe. A substrate is placed at $365 \mathrm{~mm}$ above the source plate. Several compound vacuum gauges are installed on the chamber surface to measure the local pressures.

The transport of the vapor atoms from evaporation sources is very critical to the quality of the deposited thin film. Three-dimensional DSMC simulations have helped to understand this process. In DSMC simulations, the collision parameters for metal vapors are obtained by extrapolating calculated values of other metal vapors whose viscosity coefficients are known. The DSMC results are found in excellent agreement with our measurements, such as evaporation rates of yttrium and titanium measured in-situ by quartz crystal resonators, deposited film thickness distribution measured by Rutherford backscattering spectrometer (RBS), and surface profilometer and deposited film molar ratio distribution measured by RBS and inductively coupled plasma atomic emission spectrometer (ICPAES). When the pressure in the chamber is low enough, the inter-molecular collisions can be neglected and thus the free-molecular assumption can be adopted. It is found that the free molecular assumption can be employed if particles encounter less than one collision on average when moving from an evaporative source to a substrate, which is verified by the experimental data and DSMC results.
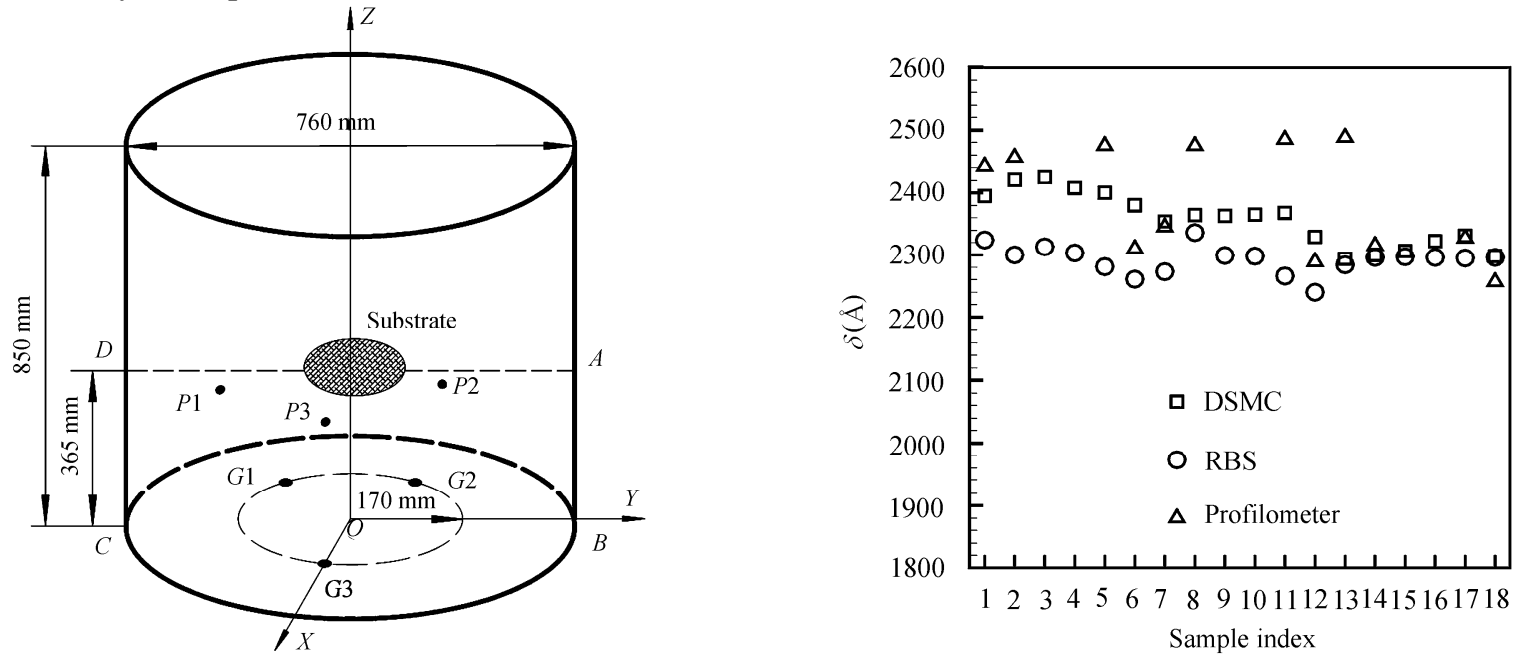

FIGURE 3. Illustration of the physical vapor deposition system (left) and comparison of film thickness obtained from DSMC simulation, spectrometer, and profilometer (right).

\section{STUDIES OF HYPERSONIC TRANSITIONAL FLOWS}

For traditional atmospheric flows, the aerodynamics of an aircraft or the thermal load on a spacecraft is always investigated using the DSMC method when the flight altitude is above $70 \mathrm{~km}$. Our extensive DSMC simulations show that the aerodynamic performance of an aircraft decreases with an increasing flight altitude: 1) the shock thickness increases and the interaction between shock wave and boundary layer is strengthened; 2) the ratio of lift to drag decreases; 3) and the relative heat transfer rate increases.

The DSMC method has also been applied to investigate the discharging process of residual rocket propellants [13]. Liquid propellant will break into droplets once it enters the space under some conditions. The droplets will 
then diffuse in the high vacuum by evaporating gas molecules. Then the freezing droplets or the vapor molecules may hit the rocket surface or other space unit, which may disturb the altitude control of the rocket and erode the space unit surface. Figure 4 shows the pressure distribution on the rocket surface, which reveals that the pressure at some locations is relatively large. Our simulated results predicted the disturbance moment of discharging propellant acting on a rocket before its launch, and they agreed with the remotely measured data in flight [13].
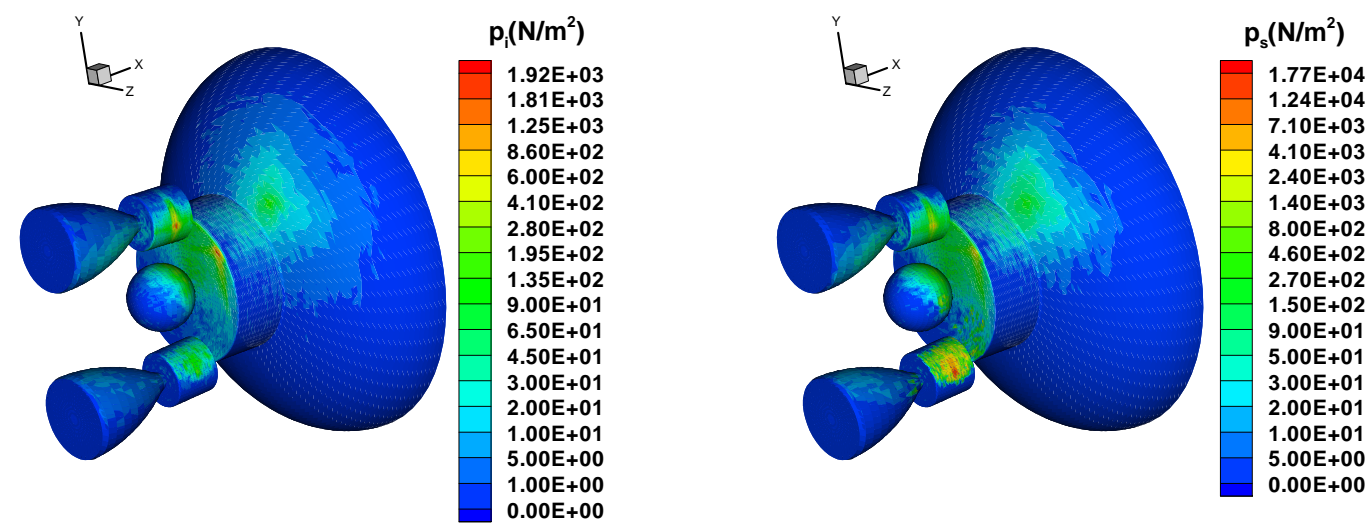

FIGURE 4. Simulated pressure distributions over a rocket surface during the discharging process of residual rocket propellants. Left: incident pressure; right: total pressure.

\section{KINETIC BEHAVIOR OF SOME CLASSICAL FLOWS}

Kinetic study of gas flows is not only important for many practical applications. It is also useful to understand the microscopic behavior of some classical problems in fluid mechanics. For instance, the Rayleigh-Bénard convection is a well-known problem where convection occurs when the Rayleigh number exceeds the critical value. Another case is the shock wave that is always treated as jump discontinuity in the continuum description.

\section{Particle Simulations of Rayleigh-Bénard Convection}

The onset of convection in an initially quiescent fluid confined between two parallel horizontal walls and heated from below is a classical problem known in hydrodynamics as the Rayleigh-Bénard (R-B) flow [14]. It is often taken as a standard model to analyze hydrodynamic instability, self-organization and transition to turbulence in fluids [15-18]. Compared to enormous works on R-B flows in the continuum viewpoint during more than one century, kinetic studies in this aspect are at a young stage. Garcia [19] firstly applied the DSMC method to the R-B flow. After many efforts [20-23], the DSMC method was extended to calculate the chaotic fluid motions at large Rayleigh (Ra) numbers [24], and the DSMC results compared well with those given by the finite difference method solving the Navier-Stokes equations.

A R-B transition from thermal conduction to convection occurs very quickly in a macroscopic sense, but it is considerably long in a microscopic sense that lasts about $10^{4}$ mean collision times at $\mathrm{Ra}=3190$. To resolve clearly and efficiently the evolution of the R-B flow patterns at low Mach number, the information preservation (IP) method is employed here. The information velocity and temperature are updated using the Octant Flux Splitting (OFS) model developed by Masters \& Ye based on the Maxwell transport equation suggested by Sun \& Boyd.
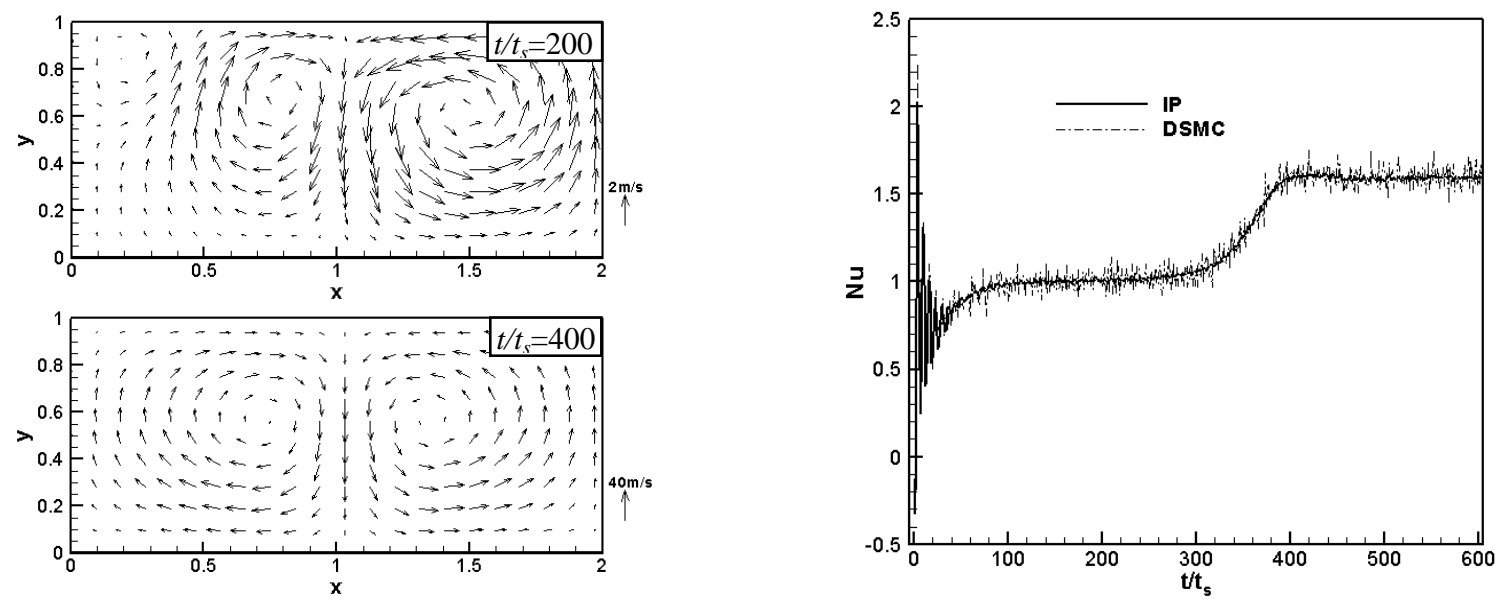

FIGURE 5. Evolution of the Rayleigh-Bénard flow at Ra=3190. Left: velocity; right: Nusselt number. 
Statistical noise inherent in particle approaches such as the direct simulation Monte Carlo (DSMC) method is effectively reduced by the IP method, and therefore the evolutions from an initial quiescent fluid to a final steady state can be shown clearly (Fig. 5).

An interesting phenomenon has been observed [25]: when the Rayleigh number (Ra) exceeds its critical value (about 1680), there exists an obvious incubation stage. During the incubation stage, the vortex structure clearly appears and evolves, whereas the Nusselt number $(\mathrm{Nu})$ of the lower plate is close to unity. After the incubation stage, the vortex velocity and Nu rapidly increase, and the flow field quickly reaches a steady, convective state. A relation of Nu to Ra given by IP agrees with those given by DSMC, the classical theory and experimental data.

The transition from thermal conduction to convection is a development process, which means that something in the inital state must relate to the final convection. In order to understand this connection, the density fluctuations in the flow is investigated using the DSMC method [26]. The particle simulation results show a clear connection between the static correlation functions of fluctuations below the critical Rayleigh number and the flow patterns above the onset of convection for small Knudsen number flows ( $\mathrm{Kn}=0.01$ and $\mathrm{Kn}=0.005)$. For a large Knudsen number, the fluctuation correlations in the horizontal direction are negligible, and the thermal conductive state is always stable. These observations suggest that the characteristics of the fluctuations below the onset of thermal instability can be used to decide whether there will be convection and to determine the possible flow patterns when convection appears.

\section{Nonequilibrium Solutions of Shock Wave and Contact Discontinuity}

Shock waves and contact discontinuities are mathematical discontinuities only for the equilibrium hyperbolic equations, where there are no particle collision time and macroscopic dissipative time scales. The discontinuity jumps have been used extensively as benchmark solutions for the development of computational fluid dynamics (CFD) algorithms. However, with the further extension of the CFD method to non-equilibrium flows, and the intensive research projects related to the multiscale modeling and simulation, it will be helpful to provide a few physical non-equilibrium solutions in order to validate new physical models and algorithms. The studies of contact wave and shock structures are two excellent examples.

The DSMC method is employed to study the unsteady behavior of both shock wave and contact discontinuity from a simple mathematical jump to a well-defined dissipative structure. The temporal and length scales are in the order of magnitude of the molecular mean collision time and mean free path. Particle simulations show that shock waves behave differently from contact discontinuities (Fig. 6). Contact discontinuities diffuse at the mean thermal speed during an early time, then slow down to a pace that the thickness of the contact discontinuities is proportional to the square root of the time. Shock waves, on the other hand, reach gradually to a steady state because the characteristic lines from each side of a shock compress to have a mechanism to prevent further diffusion of the wave. This process is rather quick since it only takes about several mean collision times for a strong shock wave to reach the steady state. Because contact discontinuities keep diffusing, it may not be appropriate to be treated as jump discontinuities for cases where the discontinuities exist for a long time. Shock waves, on the other hand, can be regarded as jump discontinuities as long as the shock strength is not very weak.
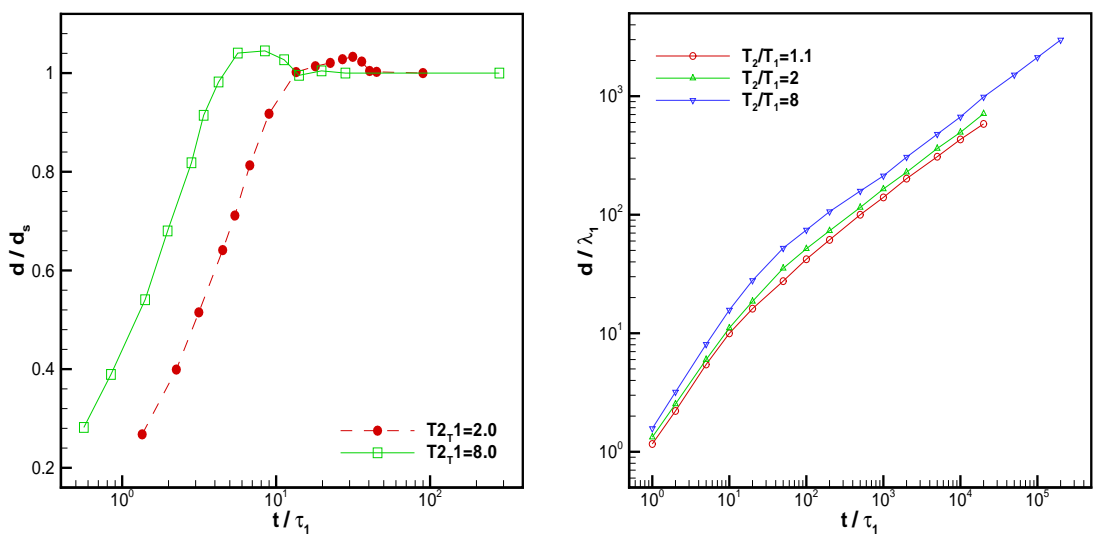

FIGURE 6. Time evolution of the thickness of contact discontinuities (left) and shock waves (right).

\section{CONCLUSIONS}

Studies on kinetic effects on gas flows were very active during the fifties of the last century and are getting revived these years. This is because the needs from aerospace and other industries are becoming increased and simulation techniques are improved to pace up with the needs. In general, gas flows under extreme conditions such as low pressure and small size conditions usually involve kinetic effects. Studies of these flows mainly depend on numerical simulations that are based on the Boltzmann equation or microscopic motions of particles. Simulations are 
usually numerical expensive, but the increasing CPU speed and parallel techniques are obviously the other factors for the current success. Finally, the microscopic mechanism of classical fluid mechanics seems to be a potential field to be studied kinetically.

\section{Acknowledgments}

The authors would like to thank our colleagues and students in our group for their research. The contents presented in this paper were partially supported by the Research Grants from National Natural Science Foundation of China (Grants No. 90205024, No. 10621202, No. 90816012, No. 50836007, and No. 10742001).

\section{References}

1. T. Abe (Ed.), Rarefied gas dynamics: 26th international symposium on rarefied gas dynamics, AIP Conference Proceedings 1084, American Institute of Physics, 2009.

2. C. Cercignani, Rarefied gas dynamics, Cambridge Texts in Applied Mathematics, Cambridge University Press, 2000.

3. G.A. Bird, Molecular Gas Dynamics and the Direct Simulation of Gas Flows, Clarendon Press, 1994.

4. J. Fan and C. Shen, "Statistical simulation of low-speed unidirectional flows in transition regime", in Rarefied gas dynamics, edited by Brun et al., Cepadus-Editions, Toulouse, Vol. 2, p. 245-252, (1999); "Statistical simulation of low-speed rarefied gas flows”, J. Comput. Phys., 167, 393-412 (2001).

5. C. Shen, J. Fan, and C. Xie, "Statistical simulation of rarefied gas flows in microchannels", J. Comput. Phys. 189, $512-526$ (2003).

6. Q. Sun and I.D. Boyd, "Drag on a flat plate in low-Reynolds number gas flows", AIAA J., 42, 1066-1072 (2004); "Flat plate aerodynamics at very low Reynolds numbers”, J. Fluid Mech., 502, 199-206 (2004).

7. C.P. Cai, I.D. Boyd, J. Fan, G.V. Candler, "Direct simulation methods for low-speed microchannel flows", Journal of Thermophysics and Heat Transfer, 14: 368-378 (2000).

8. Q. Sun, I.D. Boyd, “A direct simulation method for subsonic, micro-scale gas flows”, J. Comput. Phys., 179(2), 400-425 (2002).

9. J. Li, C. Shen, and J. Fan, "IP simulation of micro gas flows under 3-D head sliders”, in Rarefied Gas Dynamics, edited by Abe T., 1003-1008 (2009).

10. Q. Sun, I.D. Boyd, “Theoretical development of the information preservation method for strongly nonequilibrium gas flows", AIAA 2005-4828 (2005).

11. N.D. Masters and W. Ye, “Octant flux splitting information preservation DSMC method for thermally driven flows”, J. Comput. Phys., 226(2), 2044-2062 (2007).

12. S. Li, Y. Shu and J. Fan, "Thickness and component distributions of yttrium-titanium alloy films in electron-beam physical vapor deposition”, Science in China Series E: Technological Sciences, 51(9), 1470-1482 (2008).

13. J. Fan, H. Liu, J. Jiang, S. Peng and C. Shen, “Analysis and simulation of discharging residual rocket propellants in orbit” (in Chinese), ACTA Mechanica Sinica, 36(2), 129-139 (2004).

14. S. Chandrasekhar, Hydrodynamic and Hydromagnetic Stability, Clarendon, Oxford, 1961.

15. E. L. Koschmieder, Bénard cells and Taylor vortices, Cambridge University Press, Cambridge, 1993.

16. R. P. Behringer, “Rayleigh- Bénard convection and turbulence in liquid helium”, Rev. Mod. Phys. 57, 657 (1985).

17. M. C. Cross and P.C. Hohenberg, "Pattern formation out of equilibrium”, Rev. Mod. Phys. 65, 851 (1993).

18. E. Bodenschatz, W. Pesch and G. Ahlers, "Recent developments in Rayleigh-Bénard convection”, Annu. Rev. Fluid Mech. 32, 709 (2000).

19. A. Garcia, "Hydrodynamic fluctuations and the direct simulation Monte Carlo method", in Microscopic Simulation of Complex Flows, edited by M. Mareschal, Plenum, New York, 1990, pp. 177-188.

20. A. Garcia and C. Penland, "Fluctuating hydrodynamics and principal oscillation pattern analysis”, J. Stat. Phys. 64, 1121 (1991).

21. E. Golshtein and T. Elperin, “Convective instabilities in rarefied gases by direct simulation Monte Carlo method”, $J$. Thermophys. Heat Transfer, 10, 250 (1996).

22. T. Watanabe, H. Kaburaki and M. Yokokawa, "Simulation of a two dimensional Rayleigh-Bénard system using the direct simulation Monte Carlo method”, Phys. Rev. E, 49, 4060 (1994).

23. S. Stefanov and C. Cercignani, "Monte Carlo simulation of Bénard’s instability in a rarefied gas”, Eur. J. Mech. B/Fluids, 11, 543 (1992).

24. S. Stefanov, V. Roussinov, and C. Cercignani, “Rayleigh-Bénard flow of a rarefied gas and its attractors”, Phys. Fluids, 14, 2255 (2002).

25. J. Zhang and J. Fan, "Information preservation modelling of Rayleigh-Bénard transition from thermal conduction to convection”, in Rarefied Gas Dynamics, edited by Abe T., Kyoto, Japan, 359-364 (2009).

26. J. Zhang and J. Fan, "Monte Carlo simulation of thermal fluctuations below the onset of Rayleigh-Bénard convection", Physical Review E, 79 (5), 056302 (2009). 